\title{
Corticosteroids for the management of parapneumonic pleural effusion in children: a 15-year experience
}

\author{
MATTHIEU THIMMESCH ${ }^{1}$, Frédéric LEBRUN ${ }^{2}$, Frédéric PIERART ${ }^{2}$, Caroline GENIN ${ }^{2}$, \\ Isabelle LOECKX ${ }^{2}$, André MULDER ${ }^{3}$, and Pierre DEMARET ${ }^{1}$
}

${ }^{1} \mathrm{CHC}$ MontLégia

${ }^{2}$ CHC MontLégia

${ }^{3}$ MontLégia

March 20, 2021

\begin{abstract}
Objective: Description of the use of corticosteroids for the management of parapneumonic pleural effusion in children. Methods: Retrospective monocenter cohort study of all children hospitalized with a discharge diagnosis of parapneumonic pleural effusion during a 15-year period. Results: We documented 97 cases of parapneumonic effusion during the study period, with a median age (interquartile range (IQR)) of 43 (33-61) months. Most of the children benefited from an evacuation of the pleural effusion (89/97, 91.8\%): 21 patients $(21.6 \%)$ were treated with needle thoracocentesis only, while a chest tube was inserted in 68 children $(70.1 \%)$. Thirty-two patients $(33 \%)$ were treated with intrapleural fibrinolysis. Fifty-five children (56.7\%) received corticosteroids for persistent fever. The median time (IQR) between hospital admission and initiation of corticosteroids was 5.5 (4-7) days. When corticosteroids were initiated, children were febrile since 9 (IQR 8-11) days. The fever ceased in a median (IQR) of 0 (0-1) day after corticosteroids initiation. Only 1 patient required a video-assisted thoracoscopy that was provided because of morphological reasons (morbid obesity). No children treated with corticosteroids required surgery. All children were discharged alive from hospital. The median (IQR) hospital length of stay was 11 (8-14) days, with no difference between children with and without corticosteroids. Conclusion: Our results indicate that corticosteroids could be associated with a significant reduction in the use of surgical procedures and with a prompt clinical improvement. Corticosteroids could thus offer a non-invasive therapeutic alternative for children with parapnemonic effusions when antibiotics and pleural drainage are considered a failure.
\end{abstract}

\section{Introduction}

Pediatric community-acquired pneumonia (CAP) is a significant health problem and a leading infectious cause of childhood morbidity and mortality worldwide ${ }^{1}$. Even though the once significant mortality rates associated with CAP have decreased in the industrialized world thanks to vaccines, accessible antibiotics and advances in diagnostics and monitoring, some complications are still a matter of concern, most commonly parapneumonic effusions that have been increasing in Europe and the United States during the last decades ${ }^{2}$.

Parapneumonic effusion complicates up to $13 \%$ of cases of hospitalized pneumonia among children ${ }^{3}$. It can be divided into three stages: inflammatory exudate, clear in appearance, sterile (stage I); purulent exudate resulting from translocation of white blood cells into the fluid with deposition of fibrin in the pleural space (stage II); organized empyema with formation of a thick membrane covering the visceral pleura (stage III) 4,5. The management of parapneumonic effusions remains an area of controversy, and various therapeutic options are described ${ }^{6,7}$. If conservative treatment with antibiotics alone is a reasonable option for small parapneumonic effusions, guidelines recommend evacuation of effusions which are enlarging, compromising respiratory function and/or associated with loculations; intrapleural fibrinolysis is usually suggested for 
effusions of thick fluid with loculations or empyema; and surgery (either thoracotomy with decortication or video-assisted thoracoscopic surgery (VATS)) is recommended in case of failure of antibiotics, chest tube drainage and fibrinolytics ${ }^{8,9}$. However, surgery is invasive and not without risks, requires general anesthesia as well as a highly skilled pediatric surgical team, and is more expensive than conservative management ${ }^{10}$. Therefore, alternative non-surgical strategies would be appealing in children failing antibiotics, drainage and intrapleural fibrinolysis.

Inflammation plays a key role in the genesis of parapneumonic effusion ${ }^{11}$. Corticosteroids have well-known anti-inflammatory properties, based on which they have been successfully studied in several infectious diseases including bacterial meningitis and community-acquired pneumonia of the adult ${ }^{12,13}$. However, even though their use may be attractive, corticosteroids are not included in the recommendations for the management of complicated parapneumonic effusion, probably because evidence to support this approach is still lacking. Despite this, aiming to favor a less invasive approach than surgery, we have introduced corticosteroids in the therapeutic armamentarium used in managing children with complicated parapneumonic effusion admitted to our hospital. We prescribe corticosteroids to children with persisting fever despite pleural drainage and fibrinolysis, or to febrile children with effusions that are organized and loculated to such an extent that chest drainage is not an option. We report here our 15-year experience with this strategy.

\section{Methods}

Study design, study site and population

This is a 15-year monocenter retrospective study conducted in the pediatric department of a community general hospital in Liège, Belgium. Approximately 3000 children are hospitalized each year in our 85-bed pediatric department for numerous medical or surgical reasons (with the exclusion of organ transplantation and cardiac surgery).

We retrospectively searched for children (0-15-years-old) admitted to our pediatric department between January $1^{\text {st }}, 2002$, and June 30st, 2017 and coded in the hospital database with the diagnosis of pleural effusion, pleurisy or empyema. We also listed all the children for whom a chest drainage system (PleurEvac@) had been delivered by the hospital pharmacy during the study period. The medical charts of the selected children were reviewed, and only children with pleural effusion in the context of an infectious respiratory disease (as documented in the medical chart) were retained for analysis.

Data collection

Data were retrospectively extracted from medical charts by three investigators (M.T., F.L. and P.D.). Baseline data included medical history, age, main complaints and clinical findings at hospital admission. Tachycardia was defined according to the age based on the following thresholds: 180 beat per minute (bpm) for infants, and $160 \mathrm{bpm}$ for children aged 1-16-years. The increased work of breathing was documented as per medical notes, based on a subjective assessment by the treating physician (no scale measurement).

Data collected during the hospital stay included the use of at least one dose of corticosteroid (prednisone or methylprednisolone) as part of the management of the pleural effusion, the use of respiratory support techniques (high-flow oxygen therapy, noninvasive ventilation, mechanical ventilation), the number of chest imaging exams and the use of painkillers. We also collected data to characterize the pleural effusion, including the time of diagnosis, the presence of fibrin in the effusion (according to the chest ultrasound protocol), the time and type (needle thoracocentesis, chest tube) of evacuation (if any), the duration of drainage (if any), the total volume drained, the use of intrapleural fibrinolysis, as well as the leucocyte count of the pleural effusion. We also collected the total duration of fever (defined as a temperature [?] 38degC), the highest C-reactive protein (CRP) level as well as the highest leukocyte count. Finally, we recorded the duration of the hospital stay.

Statistical analyses

Descriptive statistics were reported as frequencies and percentages or as median (interquartile range). Sta- 
tistical analyses were conducted to compare children with and without corticosteroids: continuous variables were compared using Student $t$ test if normally distributed (as attested by the Shapiro-Wilk test) or Wilcoxon test if not, and the chi-square statistic was used for categorical variables. Results were considered statistically significant when the $p$ value was less than 0.05 .

\section{Results}

We documented 97 cases of parapneumonic effusion admitted to our hospital during the study period. Fiftyfive of them $(56.7 \%)$ received corticosteroids.

\section{Baseline characteristics}

The median age (IQR) at hospital admission was 43 (33-61) months for the whole cohort, with no difference between children who were going to receive corticosteroids or not $(\mathrm{p}=0.667)$ (Table 1). Most of the included children were previously healthy, even though $27.8 \%$ reported a previous respiratory disease. The main complaints at hospital admission were fever (97.9\%), asthenia (82.5\%), cough (67\%), dyspnea (39.2\%), abdominal pain $(39.2 \%)$ and chest pain $(23.7 \%)$, with no difference between children about to receive corticosteroids or not. Only a minority of children showed tachycardia at hospital admission (13.7\%), and oxygen saturation at baseline was normal for the majority of the children (median (IQR) 96 (94-98) \% for the whole cohort) with no difference for children about to receive corticosteroids or not $(\mathrm{p}=0.341)$.

\section{Corticosteroids use}

Methylprednisolone was used for each of the 55 children with corticosteroids, at an initial median (IQR) dose of $2(1.9-2) \mathrm{mg} / \mathrm{kg} /$ day. The median time (IQR) between hospital admission and initiation of corticosteroids was 5.5 (4-7) days. When corticosteroids were initiated, children were febrile since 9 (IQR 8-11) days. The fever ceased in a median (IQR) of $0(0-1)$ day after corticosteroids initiation. Three patients required more than 1 course of corticosteroids because of fever recurrence when corticosteroids were ceased. The median duration (IQR) of corticosteroid therapy was 8.5 (4-13.3) days.

Pleural effusion

Most of the children benefited from an evacuation of the pleural effusion (89/97, 91.8\%): 21 patients (21.6\%) were treated with needle thoracocentesis only, while a chest tube was inserted in 68 children (70.1\%) (Table 2 ). Chest tube insertion and intrapleural fibrinolysis occurred less frequently in children about to receive corticosteroids (chest tube $61.8 \%$ versus $81.0 \%, \mathrm{p}=0.041$; intrapleural fibrinolysis $25.5 \%$ versus $42.9 \%, \mathrm{p}=0.07$ ). The duration of chest drainage did not differ between children with and without corticosteroids (median (IQR) 5 (3-8.25) versus $5(3-6), \mathrm{p}=0.184)$. The total volume evacuated through the chest tube tended to be higher for children without corticosteroids (median (IQR) 480 (300-805) $\mathrm{mL}$ versus 390 (137-579) $\mathrm{mL}$ ), this discrepancy almost reaching the statistical significance threshold $(\mathrm{p}=0.07)$.

Biochemical analyses of the pleural effusion were inconstant and their results did not differ between children about to receive corticosteroids or not: proteins were measured in 79/89 samples (88.8\%) (median (IQR) 45 (39.8-48.7) g/L for the whole cohort), LDH in 64 (71.9\%) (median (IQR) 3124 (879-9367)U/L), glucose in 57 $(64 \%)$ (median (IQR) $0.22(0.01-0.67) \mathrm{g} / \mathrm{L})$, amylase in $15(16.9 \%)$ and $\mathrm{pH}$ in only 8 samples $(9 \%)$. Cytology was more frequently analyzed: the leucocyte and the red blood cell counts were requested in $78(87.6 \%)$ and in $73(82 \%)$ samples respectively and they did not differ between children with and without corticosteroids (median (IQR) 5200 (1000-17800) red blood cells /mm3 and 6800 (1500-21100) white blood cells /mm3 for the whole cohort).

Surgery

Only 1 patient required a VATS that was provided because of morphological reasons (morbid obesity) compromising our usual "non-surgical" chest tube insertion. This patient did not receive corticosteroids. No children treated with corticosteroids needed surgery.

Organ support 
The proportion of children admitted to the PICU / middle care during the hospital stay was lower for children receiving corticosteroids $(60.9 \%$ versus $90.5 \%, \mathrm{p}=0.011)$, and the duration of PICU / middle care stay was shorter for children with corticosteroids than for those without corticosteroids (median (IQR) 5.5 (4-9.3) versus 8.5 (5.8-14.3) days, $\mathrm{p}=0.017$ ) (Table 2 ).

Organ support therapies were rare: only 1 patient (1\%) received high-flow oxygen therapy, no patient received noninvasive ventilation, 4 patients $(4.1 \%)$ were mechanically ventilated and 2 patients $(2.1 \%)$ required inotropic and vasopressive support.

Medical imaging

The number of chest X-rays was lower for children with corticosteroids than for those without (median (IQR) $2(2-4)$ versus $4(2-6), \mathrm{p}=0.01)$, while the number of chest ultrasounds and chest computed tomography scans did not differ between the 2 groups.

\section{Inflammation}

The total duration of fever was 10 (8-13) days for the whole cohort, with no difference between children with and without corticosteroids. There was a marked inflammatory response as assessed by a high CRP level (median peak (IQR) $270(202-370) \mathrm{mg} / \mathrm{L}$ ) and an increased leukocyte count (median peak (IQR) 20200 (16700-27200) leukocytes /mm3), with no difference between children with and without corticosteroids.

Pain control

All but one child received paracetamol during the hospital stay, most of children received ibuprofen (95.9\%), and $76(78.4 \%)$ children received at least one dose of tramadol. Morphine was prescribed to $8(8.2 \%)$ children. The use of painkillers did not differ between children with and without corticosteroids.

\section{Outcome}

All children were discharged alive from hospital: 92 children were discharged home, and 5 were discharged to another hospital (the referring hospital). The median (IQR) hospital length of stay (for children discharged home) was 11 (8-14) days, with no difference between children with and without corticosteroids.

\section{Discussion}

In our cohort of 97 children with parapneumonic effusion, $55(56.7 \%)$ received corticosteroids because of refractory fever. The fever ceased within the day following corticosteroids initiation for most of the treated children and none of them required subsequent surgery. Hospital stay characteristics and outcomes were the same for children with and without corticosteroids. All the children were discharged alive and well from hospital.

Recommended management of pediatric parapneumonic effusions

The treatment of choice for parapneumonic effusion in children remains a matter of controversy in the literature and in clinical practice, and the management of this disease remains largely center dependent ${ }^{14}$.

All children with parapneumonic effusion should be admitted to the hospital ${ }^{8}$. The majority of the children included in our study were hospitalized in PICU / middle care. However, contrary to adult empyema, parapneumonic effusions in childhood are associated with a low mortality and the vast majority of children recover completely ${ }^{15}$. The high admission rate to PICU in our cohort is explained by our admission policies rather than by the severity of the disease.

Although it has been reported than approximately 20 to $50 \%$ of the parapneumonic effusions can be treated with intravenous antibiotics alone, there is some evidence that early evacuation of the pleural effusion may be beneficial ${ }^{16,17}$. The role of needle thoracocentesis in the management of pediatric parapneumonic effusions remains unclear, and some advocate that it should be reserved for cases where non-infective causes of parapneumonic effusions are being considered ${ }^{15}$. However, needle aspirations have advantages: it less invasive than a chest tube, it is relatively easy and safe to perform in older children who can tolerate 
the procedure with a local anesthetic and sedation, and the sample collected via this minimally invasive procedure can be analyzed for diagnostic clarifications. Therefore, a single simple needle thoracocentesis may be considered for the management of some free-flowing parapneumonic effusion. However, repeated taps are not recommended, and a drain should be inserted once it is clear a second tap is required; moreover, chest tube is recommended over simple thoracocentesis for loculated and/or purulent effusions ${ }^{8,9}$. In our cohort, one fifth $(21.6 \%)$ of the children were successfully managed with simple needle thoracocentesis, confirming that this less-invasive strategy may be appropriate for selected cases.

In a large cohort study including 14225 pediatric empyema-related hospital discharges aiming to describe trends in the number and type of empyema-related procedures in the United States between 2008 and 2014, there was an increase in the proportions of patients coded for chest tube placement as the only procedure (from $14.6 \%$ to $20.9 \%$ ), for 2 chest tube procedures (from $0.9 \%$ to $3.5 \%$ ), and for chest tube placement as part of their management along with other therapeutic strategies (from $24.8 \%$ to $30.6 \%$ ) ${ }^{18}$. In another cohort of 192 Australian children with a diagnosis of empyema, $174(90.6 \%)$ were managed by primary chest drainage, among which 49 (28.1\%) required subsequent VATS. In this study, the authors failed to identify risk markers of VATS at the time of insertion of the primary chest tube, which highlights the difficulties in identifying patients at risk of drainage failure. They observed, however, that each day of fever after the chest tube insertion was associated with an increased risk of VATS ${ }^{19}$. Our results are within the range suggested by these 2 recent studies: $70.1 \%$ of the children included in our study were managed with a chest tube. Such discrepancies between the reported proportion of primary chest drainage may be explained by differences in patient characteristics, as well as differences in management strategies since the priority of the available therapeutic interventions is not clearly defined.

Pediatric data on intrapleural fibrinolysis are scarce, but indicate that instillation of a fibrinolytic agent through the chest tube may be of interest by reducing the hospital length of stay ${ }^{20}$. Therefore, intrapleural fibrinolysis is recommended for parapneumonic effusions of thick fluid with loculations and/or empyema (overt pus) ${ }^{8}$. The agent, dosage, concentration, dwell time, interval and total doses have not been proven with comparative data and are subject for future investigation ${ }^{9}$. In our cohort, only $32 / 68$ (47.1\%) of the children with a chest tube received intrapleural fibrinolysis, while fibrin was found in the pleural ultrasound of $77.7 \%$ of the children and while the median leucocyte count (IQR) of $6.8(1.5-21.1) \times 10^{3} / \mathrm{mm}^{3}$ indicates that most of the effusions were purulent. Our practice is thus not totally in accordance with the aforementioned recommendation, maybe because the level of evidence for this recommendation is weak.

Primary VATS is associated with good clinical outcomes and low rates of reintervention ${ }^{19}$. A recent metaanalysis suggested that VATS may be associated with a lower reintervention rate and shorter post-procedural length of stay than primary chest tube and fibrinolysis ${ }^{7}$. However, the level of evidence is low, and the authors of this meta-analysis acknowledge that chest drainage with fibrinolysis may be effective in more than $75 \%$ of cases. Furthermore, VATS require minimally invasive surgery expertise which is not routinely available. The place of VATS in the management of parapneumonic effusions in children is thus not clearly defined. In our cohort, only 1 child (1\% of the whole cohort) was treated with VATS. This only VATS was required because insertion of a chest tube at the bedside was technically challenging (morbid obesity). No child received VATS because of a failure of non-surgical management. This proportion of children requiring surgery is remarkably low, considering that others have reported that the need for surgical rescue interventions for parapneumonic empyema in children remained as high as $20 \%^{21}$. Our finding is probably explained by the use of corticosteroids if chest drainage was deemed not possible (multiloculated effusion not drainable) or as soon as chest drainage was considered a failure (persisting fever).

Corticosteroids for the management of pediatric parapneumonic effusions

In a randomized controlled trial conducted in Spain and including 60 children hospitalized with community acquired pneumonia and pleural effusion, the use of dexamethasone $(0.25 \mathrm{mg} / \mathrm{kg} / 6$ hours for 48 hours $)$ versus placebo reduced the time to recovery by almost 3 days with no apparent increase in serious adverse events. In that study, more patients receiving placebo than receiving dexamethasone needed eventual pleural drainage $(16 \%$ versus $5 \%)$, but the study was underpowered to determine statistical significance for this analysis ${ }^{22}$. 
To date, no other studies have assessed the role of corticosteroids in the management of parapneumonic effusion in children. However, this question makes sense, especially when the inflammatory response is clinically evident. Indeed, the anti-inflammatory properties of corticosteroids no longer need to be demonstrated ${ }^{23}$. Beneficial effects of corticosteroids have been observed in several infectious processes where inflammation may play a key role, including respiratory diseases: in patients hospitalized with community-acquired pneumonia, the use of (methyl)prednisone reduces treatment failure and improves recovery, and meta-analysis of the few available randomized trials suggest that corticosteroids are associated with a reduction in mortality, in need for mechanical ventilation and in hospital length of stay; moreover, the use of dexamethasone in patients hospitalized with coronavirus disease 2019 (COVID-19) recently resulted in lower 28-day mortality among those receiving respiratory support ${ }^{13,24-26}$. But corticosteroids have also potential drawbacks, as they can cause systemic immunosuppression ${ }^{27}$. Corticosteroid treatment in influenza is associated with increased mortality and hospital-acquired infection, even though the evidence relates mainly to high corticosteroid doses and is of low quality with a high potential for confounding by indication ${ }^{28}$.

Considering the lack of evidence, our approach is rather cautious and conservative: we use corticosteroids as a rescue therapy when antibiotics and pleural drainage are considered a failure, only after a few days of intravenous antibiotics and only if no lung abscess has been found on chest tomodensitometry. With this strategy, no children underwent surgery in our cohort for failure of the medical management.

Limitations and strengths

Our study has several limitations. First, it is single center, which limits its external validity. Second, the retrospective design increases the risk of information bias. Third, the use of corticosteroids was not strictly protocolized so that the criteria for initiating corticosteroids may have varied from one child to another. Fourth, we only collected data during the hospital stay: therefore, we cannot exclude that corticosteroids are associated with worse outcomes after hospital discharge. However, even though children with parapneumonic effusions may have severe clinical, radiological and lung function abnormalities during the acute phase of the disease, favorable and complete recovery is the rule in the months following infection ${ }^{29,30}$. Therefore, the benefits of corticosteroids should be expected during the hospital stay or maybe in the immediate posthospital phase, but not in the long term. Fifth, microbiological, clinical and management changes may have occurred during the 15-year study period, and data observed 15 years ago may not be representative of what occurs nowadays. The main strengths of our study are the size of our cohort, and most importantly

the fact that our study is the first to describe the successful use of corticosteroids in the management of parapneumonic effusion when non-surgical management is considered a failure.

\section{Conclusion}

Corticosteroids may be an important part of the therapeutic armamentarium for children with parapneumonic effusion. Obviously, lots of questions have to be answered: what molecule (if any) should be used, what posology, when to initiate the treatment (in the early phase of the disease, or later?), what duration of treatment, what patients would benefit from this treatment? As a first step in addressing these questions, our data, despite their limitations, indicate that corticosteroids could be associated with a significant reduction in the use of surgical procedures and with a prompt clinical improvement. Corticosteroids could thus offer a non-invasive therapeutic alternative for children with parapneumonic effusions when antibiotics and pleural drainage are considered ineffective.

\section{Bibliography}

1. Walker CLF, Rudan I, Liu L, Nair H, Theodoratou E, Bhutta ZA, O'Brien KL, Campbell H, Black RE. Global burden of childhood pneumonia and diarrhoea. Lancet 2013;381(9875):1405-1416.

2. Boyd K. Back to the Basics: Community-Acquired Pneumonia in Children. Pediatr Ann 2017;46(7):e257e261.

3. Jain S, Self WH, Wunderink RG, Fakhran S, Balk R, Bramley AM, Reed C, Grijalva CG, Anderson EJ, Courtney DM, et al. Community-Acquired Pneumonia Requiring Hospitalization among U.S. Adults. N 
Engl J Med 2015;373(5):415-427.

4. Santanakrishnan R, Murali GS, Javaregowda D, Shankar G, Babu N, Jadhav V. Thoracoscopy in Stage 3 empyema thoracis in children - A safe and feasible alternative to thoracotomy. J Pediatr Surg 2019.

5. Griffith D, Boal M, Rogers T. Evolution of practice in the management of parapneumonic effusion and empyema in children. J Pediatr Surg 2018;53(4):644-646.

6. Darby JB, Singh A, Quinonez R. Management of Complicated Pneumonia in Childhood: A Review of Recent Literature. Rev Recent Clin Trials 2017;12(4):253-259.

7. Pacilli M, Nataraja RM. Management of paediatric empyema by video-assisted thoracoscopic surgery (VATS) versus chest drain with fibrinolysis: Systematic review and meta-analysis. Paediatr Respir Rev 2019;30:42-48.

8. Balfour-Lynn IM, Abrahamson E, Cohen G, Hartley J, King S, Parikh D, Spencer D, Thomson AH, Urquhart D, Paediatric Pleural Diseases Subcommittee of the BTSSoCC. BTS guidelines for the management of pleural infection in children. Thorax 2005;60 Suppl 1:i1-21.

9. Islam S, Calkins CM, Goldin AB, Chen C, Downard CD, Huang EY, Cassidy L, Saito J, Blakely ML, Rangel SJ, et al. The diagnosis and management of empyema in children: a comprehensive review from the APSA Outcomes and Clinical Trials Committee. J Pediatr Surg 2012;47(11):2101-2110.

10. Paraskakis E, Vergadi E, Chatzimichael A, Bouros D. Current evidence for the management of paediatric parapneumonic effusions. Curr Med Res Opin 2012;28(7):1179-1192.

11. Marchi E, Vargas FS, Acencio MM, Sigrist RMS, Biscaro MDA, Antonangelo L, Teixeira LR, Light RW. Proinflammatory and antiinflammatory cytokine levels in complicated and noncomplicated parapneumonic pleural effusions. Chest 2012;141(1):183-189.

12. Kim KS. Acute bacterial meningitis in infants and children. Lancet Infect Dis 2010;10(1):32-42.

13. Blum CA, Nigro N, Briel M, Schuetz P, Ullmer E, Suter-Widmer I, Winzeler B, Bingisser R, Elsaesser H, Drozdov D, et al. Adjunct prednisone therapy for patients with community-acquired pneumonia: a multicentre, double-blind, randomised, placebo-controlled trial. Lancet 2015;385(9977):1511-1518.

14. Hafen GM, Grenzbach AC, Moeller A, Rochat MK. Lack of concordance in parapneumonic effusion management in children in central Europe. Pediatr Pulmonol 2016;51(4):411-417.

15. Walker W, Wheeler R, Legg J. Update on the causes, investigation and management of empyema in childhood. Arch Dis Child 2011;96(5):482-488.

16. Carter E, Waldhausen J, Zhang W, Hoffman L, Redding G. Management of children with empyema: Pleural drainage is not always necessary. Pediatr Pulmonol 2010;45(5):475-480.

17. Segerer FJ, Seeger K, Maier A, Hagemann C, Schoen C, van der Linden M, Streng A, Rose MA, Liese JG. Therapy of 645 children with parapneumonic effusion and empyema-A German nationwide surveillance study. Pediatr Pulmonol 2017;52(4):540-547.

18. Kelly MM, Coller RJ, Kohler JE, Zhao Q, Sklansky DJ, Shadman KA, Thurber A, Barreda CB, Edmonson MB. Trends in Hospital Treatment of Empyema in Children in the United States. J Pediatr 2018;202:245-251 e241.

19. Haggie S, Gunasekera H, Pandit C, Selvadurai H, Robinson P, Fitzgerald DA. Paediatric empyema: worsening disease severity and challenges identifying patients at increased risk of repeat intervention. Arch Dis Child 2020;105(9):886-890.

20. Thomson AH, Hull J, Kumar MR, Wallis C, Balfour Lynn IM. Randomised trial of intrapleural urokinase in the treatment of childhood empyema. Thorax 2002;57(4):343-347. 
21. Proesmans M, Gijsens B, Van de Wijdeven P, De Caluwe H, Verhaegen J, Lagrou K, Van Even E, Vermeulen F, De Boeck K. Clinical outcome of parapneumonic empyema in children treated according to a standardized medical treatment. Eur J Pediatr 2014;173(10):1339-1345.

22. Tagarro A, Otheo E, Baquero-Artigao F, Navarro ML, Velasco R, Ruiz M, Penin M, Moreno D, Rojo P, Madero R, et al. Dexamethasone for Parapneumonic Pleural Effusion: A Randomized, Double-Blind, Clinical Trial. J Pediatr 2017;185:117-123 e116.

23. Rhen T, Cidlowski JA. Antiinflammatory action of glucocorticoids-new mechanisms for old drugs. N Engl J Med 2005;353(16):1711-1723.

24. Torres A, Sibila O, Ferrer M, Polverino E, Menendez R, Mensa J, Gabarrus A, Sellares J, Restrepo MI, Anzueto A, et al. Effect of corticosteroids on treatment failure among hospitalized patients with severe community-acquired pneumonia and high inflammatory response: a randomized clinical trial. JAMA 2015;313(7):677-686.

25. Group RC, Horby P, Lim WS, Emberson JR, Mafham M, Bell JL, Linsell L, Staplin N, Brightling C, Ustianowski A, et al. Dexamethasone in Hospitalized Patients with Covid-19 - Preliminary Report. N Engl J Med 2020.

26. Siemieniuk RA, Meade MO, Alonso-Coello P, Briel M, Evaniew N, Prasad M, Alexander PE, Fei Y, Vandvik PO, Loeb M, et al. Corticosteroid Therapy for Patients Hospitalized With Community-Acquired Pneumonia: A Systematic Review and Meta-analysis. Ann Intern Med 2015;163(7):519-528.

27. Schacke H, Docke WD, Asadullah K. Mechanisms involved in the side effects of glucocorticoids. Pharmacol Ther 2002;96(1):23-43.

28. Lansbury LE, Rodrigo C, Leonardi-Bee J, Nguyen-Van-Tam J, Shen Lim W. Corticosteroids as Adjunctive Therapy in the Treatment of Influenza: An Updated Cochrane Systematic Review and Meta-analysis. Crit Care Med 2020;48(2):e98-e106.

29. Maffey A, Colom A, Venialgo C, Acastello E, Garrido P, Cozzani H, Eguiguren C, Teper A. Clinical, functional, and radiological outcome in children with pleural empyema. Pediatr Pulmonol 2019;54(5):525530.

30. de Benedictis FM, Carloni I, Osimani P, Cobellis G, Martino A, Lanza C, Niccoli AA, Azzari C, Skrami E, Gesuita R. Prospective evaluation of lung function in children with parapneumonic empyema. Pediatr Pulmonol 2019;54(4):421-427.

\section{Hosted file}

Table 1.pdf available at https://authorea.com/users/402683/articles/514466-corticosteroidsfor-the-management-of-parapneumonic-pleural-effusion-in-children-a-15-year-experience

\section{Hosted file}

Table 2.pdf available at https://authorea.com/users/402683/articles/514466-corticosteroidsfor-the-management-of-parapneumonic-pleural-effusion-in-children-a-15-year-experience 